\title{
Archéopages Archopages
}

Archéologie et société

45 | 2017

De la terre au pot

\section{Les transferts de techniques potières}

Les officines antiques de Bavay, Pont-sur-Sambre et Famars (Nord)

Transfers of pottery techniques. The ancient workshops of Bavay, Pont-sur-

Sambre and Famars (Nord)

Las transferencias técnicas en alfarería. Las manufacturas antiguas de Bavay, Pont-sur-Sambre y Famars (Nord)

\section{Sonja Willems et Barbara Borgers}

\section{OpenEdition \\ Journals}

Édition électronique

URL : https://journals.openedition.org/archeopages/3108

DOI : $10.4000 /$ archeopages.3108

ISSN : 2269-9872

Éditeur

INRAP - Institut national de recherches archéologiques préventives

Édition imprimée

Date de publication : 1 mars 2018

Pagination : $72-79$

ISSN : 1622-8545

Référence électronique

Sonja Willems et Barbara Borgers, "Les transferts de techniques potières 》, Archéopages [En ligne], 45 | 2017, mis en ligne le 01 janvier 2020, consulté le 04 juin 2021. URL : http://

journals.openedition.org/archeopages/3108; DOI : https://doi.org/10.4000/archeopages.3108 


\section{Les transferts de}

\section{techniques potières}

Les officines antiques de Bavay,

Pont-sur-Sambre et Famars (Nord)

Sonja Willems Inrap, UMR 7044, «ArSCAAn

Barbara Borgers Universite de Salzbourg, Departement de Geographieet Góloggie

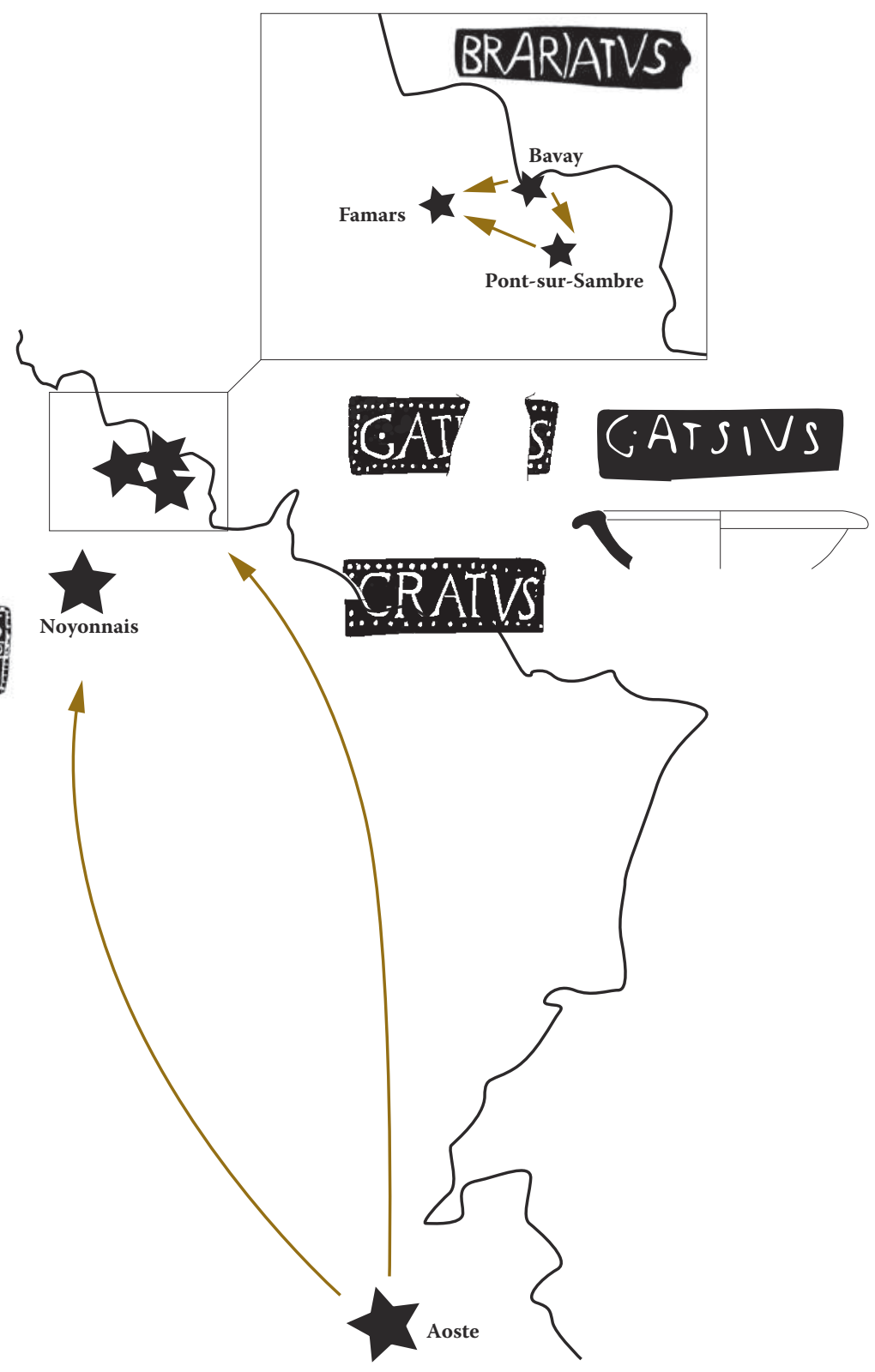

1.La présence de potiers d'Aoste dans le nord de la Gaule est attestée par la présence des estampilles des Atisii et des Valeriisur des produits des ateliers de

Noyon et de Bavay. Un mortier local de Bavay (a.), estampillé par G.ATSIVS, confirme ce transfert (fouille nouveau collège, $n^{\circ}$ Inv. 9Z735).

Le répertoire homothétique démontre clairement le lien entre les officines d'Aoste et de Bavay, aussi bien pour les mortiers, les cruches que pour les amphores régionales

(b.). Les formes évoluent

ensuite lentement et permettent de distinguer les ateliers, dès lors que les analyses chimiques et pétrographiques n'apportent plus de réponse. 
Les analyses pétrographiques et macroscopiques servent souvent en céramologie aux caractérisations et descriptions du répertoire d'une officine et de ses matières premières. Ainsi, elles facilitent l'identification de l'origine des céramiques sur les sites de consommation, résultant en cartes de distribution destinées à une meilleure compréhension des enjeux économiques. Nous avons voulu dépasser la caractérisation et tester une approche intégrée pour la compréhension des techniques potières, leur évolution chronologique et les possibles transferts de traditions. Trois officines dans la partie sud du territoire nervien se prêtent bien à l'exercice : celle de Bavay, chef-lieu de cité, et celles de Pont-sur-Sambre et de Famars, deux agglomérations secondaires, la première située à $10 \mathrm{~km}$ au sud, l'autre à $20 \mathrm{~km}$ à l'ouest de la capitale. Jusqu'à présent, les produits cuits en atmosphère oxydante (mode A selon Maurice Picon [Picon, 1973]) (cruches, mortiers, pots de stockage) des trois officines n'ont pas pu être distingués et sont rassemblés dans un grand groupe appelé « BavayFamars ». Les observations ont démontré leur importante diffusion dans le nord de la Gaule, jusqu'au limes rhénan et même en Angleterre. Ce groupe est des plus intéressants, vu sa proximité typologique et technique avec les répertoires plus méridionaux de la région d'Aoste. L'utilisation d'un traitement quasi identique des argiles calcaires dans les deux zones avait été mise en avant à partir d'une comparaison du matériel de Famars et de Lyon (Batigne-Vallet, 2001). En outre, un répertoire imité de celui d'Aoste et des estampilles des Atisii, potiers d'Aoste, ont été observés parmi le matériel produit localement à Bavay et Noyon (Dubois et al., 2009) [ill. 1].
La méthodologie et les hypothèses de travail

Dans le cadre d'une recherche doctorale sur la production des céramiques chez les Nerviens, nous avons souhaité étudier ces trois ateliers, selon deux axes de recherche. Le premier se concentre sur la question de la différenciation des trois officines. L'analyse des pâtes distingue-t-elle les productions des trois ateliers, ou prouve-t-elle leurs liens? Existe-t-il une évolution chronologique dans les techniques utilisées? Le deuxième concerne les transferts de techniques. Qui sont ces potiers? Peut-on prouver leur déménagement de Bavay vers Pont-sur-Sambre et Famars, par exemple pour le potier BRARIATVS, dont les estampilles sont retrouvées aussi bien à Bavay qu'à Pont-sur-Sambre ? Ont-ils emporté leur savoir-faire? Les matières premières locales sont-elles employées par les potiers, ou doit-on chercher d'autres sources d'approvisionnement?

Un échantillonnage a été effectué en fonction de ces thématiques de recherche. La méthode intégrée vise à combiner toutes les données disponibles (Borgers, 2015 ; Willems, Borgers, 2015). D'abord, des groupes sont définis par macroscopie au sein des lots liés à un atelier (les rebuts de production de chaque four), en prenant en compte les caractéristiques de surface, couleurs, matrices et inclusions (Willems, 2005). L'échantillonnage de chaque lot lié à un four ou à un rejet particulier aide à comprendre des différences au sein de l'officine même. Pour chaque groupe caractérisé macroscopiquement, au moins un échantillon est sélectionné par type. De cette manière, l'analyse permet de prendre en compte l'adaptation des pâtes à la catégorie et à la forme du vase. Ces échantillons servent à la fabrication de lames minces, suivant un protocole mis en place
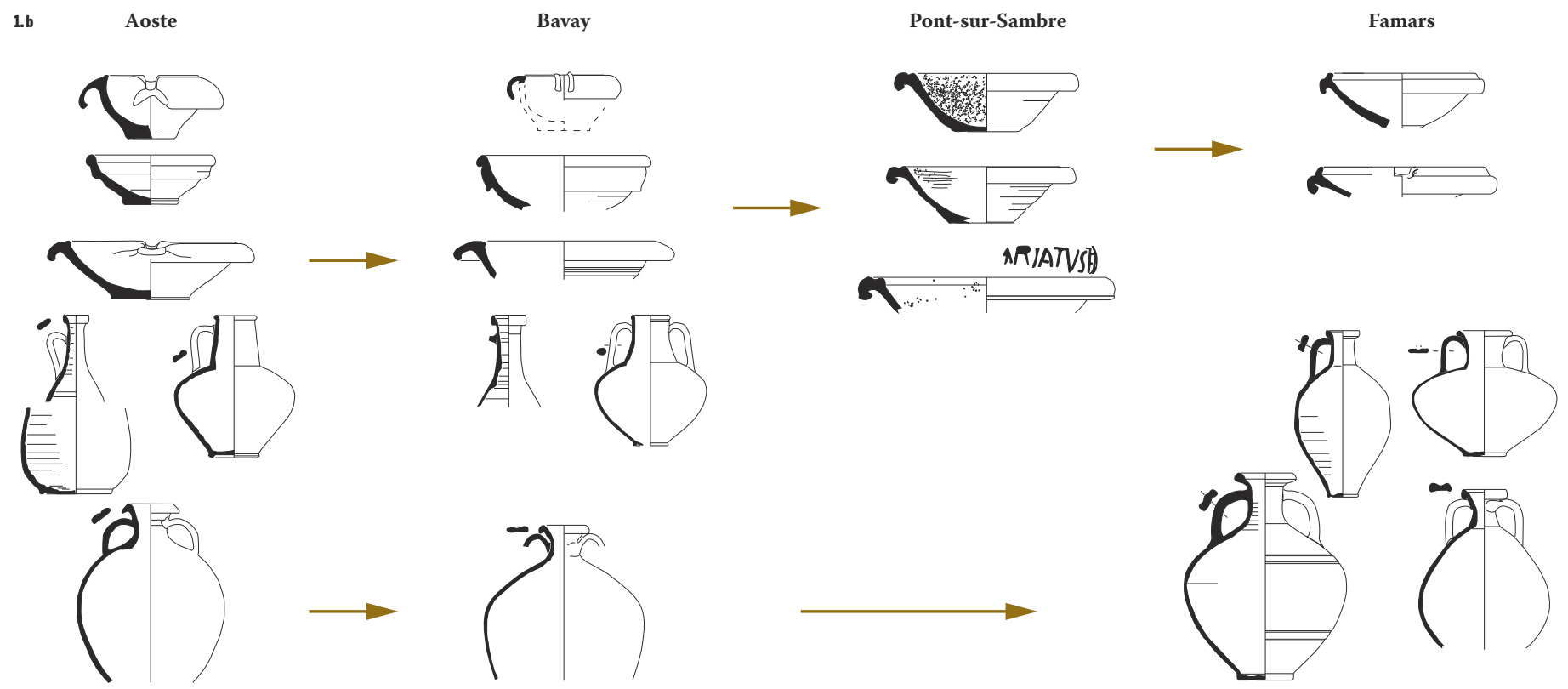


\begin{tabular}{|c|c|c|c|c|c|}
\hline Échant. & Série/échant. & Atelier/site & Formes/Argile & Structure & Pâte \\
\hline 1 & $1 / 2$ & $\begin{array}{l}\text { Famars « Technopôle } \\
\text { 2011-2014» }\end{array}$ & Cruche à 2 anses & US 1335 & Pâte 2 \\
\hline 2 & $1 / 28$ & $\begin{array}{l}\text { Famars « Technopôle } \\
\text { 2011-2014» }\end{array}$ & M1 bilobé doré au mica & US 1382 & $\begin{array}{l}\text { Variante } 1 \text { de Pâte } 2 \\
\text { (variante épurée) }\end{array}$ \\
\hline 3 & $1 / 31$ & $\begin{array}{l}\text { Famars « Technopôle } \\
\text { 2011-2014» }\end{array}$ & Cruche à 2 anses bord évasé & US 5135 & Pâte 2 \\
\hline 4 & $1 / 32$ & $\begin{array}{l}\text { Famars « Technopôle } \\
\text { 2011-2014» }\end{array}$ & Mortier à bord haut interne & US 5135 & Pâte 1 \\
\hline 5 & $1 / 33$ & $\begin{array}{l}\text { Famars « Technopôle } \\
\text { 2011-2014» }\end{array}$ & Mortier à lèvre pendante & US 5135 & Pâte 1 \\
\hline 6 & $1 / 60$ & Bavay « rue de la Gare » & Cruche & US 1054 & $\begin{array}{l}\text { Variante } 1 \text { de Pâte } 9, \\
\text { très proche de pâte } 1 \\
\text { (foraminifères) }\end{array}$ \\
\hline 7 & $1 / 61$ & Bavay « rue de la Gare » & Cruche & US 1054 & Pâte 9 \\
\hline 8 & $1 / 66$ & Bavay « rue de la Gare » & Cruche/pot doré au mica & US 1054 & Pâte 9 (foraminifères) \\
\hline 9 & $1 / 67$ & Bavay « rue de la Gare » & Cruche/pot doré au mica & US 1054 & Pâte 9 \\
\hline 10 & $1 / 72$ & Bavay « rue de la Gare » & Pot avec un bord oblique & US 1054 & $\begin{array}{l}\text { Sous-variante } 2 \text { Pâte } 9 \\
\text { (végétales brûlées) }\end{array}$ \\
\hline 11 & $1 / 73$ & Bavay « rue de la Gare » & Pot imitation de type « Besançon » & US 1054 & $\begin{array}{l}\text { Variante } 1 \text { de Pâte } 9, \\
\text { très proche de pâte } 1\end{array}$ \\
\hline 12 & $2 / 170$ & $\begin{array}{l}\text { Famars « Technopôle } \\
2013 »\end{array}$ & Mortier BAFA (UXPVRO) & 6025.1 & Pâte 16 \\
\hline 13 & $2 / 171$ & $\begin{array}{l}\text { Famars « Technopôle } \\
2013 »\end{array}$ & Mortier SAVO & Contexte III ${ }^{\mathrm{e}} \mathrm{s}$. & Pâte 16 \\
\hline 14 & $2 / 173$ & Pont-sur-Sambre 1989 & Mortier (VARIATVS) & K205 & Pâte 16 \\
\hline 15 & $2 / 174$ & Pont-sur-Sambre 1989 & Mortier (VICTOR) & K18 & Pâte 16 \\
\hline 16 & $2 / 175$ & Pont-sur-Sambre 1989 & Mortier (VARIATVS) & K227 & Pâte 16 \\
\hline 17 & $2 / 176$ & Pont-sur-Sambre 1989 & Mortier (VARIATVS) & Nr. 40 & Pâte 16 \\
\hline 18 & $2 / 177$ & Pont-sur-Sambre 1989 & Mortier (VARIATVS) & K245 & Pâte 16 \\
\hline 19 & $2 / 178$ & Pont-sur-Sambre 1989 & Mortier (BRARIATVS) & Sans nr. & $\begin{array}{l}\text { Pâte sans numéro, proche } \\
\text { d'échantillon } 16 \text { et } 21\end{array}$ \\
\hline 20 & $2 / 179$ & Bavay « Neuf Fontaines » & Mortier (ULPEFOX) & «Mouton $2 »$ & Pâte 1 \\
\hline 21 & $2 / 180$ & Bavay « Neuf Fontaines » & Mortier (BVDE) & «Mouton $2 »$ & Pâte 1 \\
\hline 22 & $2 / 181$ & Bavay « Neuf Fontaines » & Mortier (BRARIATVS) & « Mouton $2 »$ & Pâte 16 \\
\hline 23 & $2 / 182$ & Bavay « Neuf Fontaines » & Mortier (BRARIATVS) & «Mouton $2 »$ & Pâte 16 \\
\hline 24 & $2 / 185$ & Bavay « Sablière Mathieu » & $\begin{array}{l}\text { TN/TR pot tonnelet avec décor } \\
\text { incisions obliques }\end{array}$ & / & Pâte 16 \\
\hline DG01/25 & 2 & Famars & Argile tertiaire de Mont Houy & I & \\
\hline DG04/26 & 2 & Bavay & Argile tertiaire de Bellignies & 1 & \\
\hline DG05/27 & 2 & Bavay & Argile tertiaire de Bellignies & I & \\
\hline DG06/28 & 2 & Bavay & Argile tertiaire de Bellignies & 1 & \\
\hline DG07/29 & 2 & Famars & Argiles des marnes de la Rhonelle & I & \\
\hline DG08/30 & 2 & Famars & Mélange : argile tertiaire \& marnes & I & \\
\hline DG79/31 & $1 / 79$ & Famars & Argile tertiaire de Mont Houy & 1 & \\
\hline
\end{tabular}




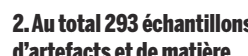

d'artefacts et de matière première, issus de différents ateliers du nord de la France, ont fait l'objet d'une analyse pétrographique, puis la moitie d'entre eux ont été étudiés chimiquement. Dans ce dernier lot, 31 échantillons ont été retenus : 24 en céramique commune cuite en mode $A$ et 7 de matière première.

. Le materiel provien du ramassage effectué lors d'aménagements à Bavay, dont le matériel a été versé au musée dan les années 1950 à 1980. Nos remerciements s'adressent à Laurent Bouthor et Véronique Mary, Forum-Musée de Bavay, qui nous ont permis l'accès aux céramiques pour

échantillonnage et étude.

2. Sous la responsabilité

de David Labarre, Inrap.

3. Merci à Patrice Herbin,

chef du service

archéologique du conseil

départemental du Nord

de nous avoir donné

accès au matériel recueilli

en 1989 à Pont-sur-

Sambre.

4. Merci à D. Labarre

pour l'argile de la carrière

et les échantillons de

la « rue de la Gare ».

5. Merci à Raphaël

Clotuche pour les argiles

du Mont Houy et les

marnes des berges de

la Rhonelle, de même

qu'à Jennifer Clerget et

Géraldine Teysseire pour

les échantillons de

Famars-Technopôle. par Patrick Quinn (Quinn, 2013). Cette deuxième étape conduit à l'identification de groupes de pâtes, reflétant des techniques et traditions de production. Un potier doit maîtriser les caractéristiques de la matière première ; il sait comment la traiter par tamisage, battage, pourquo enlever ou rajouter des dégraissants et comment la mélanger avec d'autres matières. Cette connaissance est l'aboutissement d'une période de tests qui ont conduit à la mise en place de pratiques transmises de génération en génération [cf. encadré]. Chaque pâte est la signature d'un potier ou d'un groupe de potiers, le reflet de sa volonté d'adapter la matière première en fonction de la catégorie ou de la forme finale désirée. Parmi chaque groupe pétrographique, certains échantillons ont été sélectionnés, en fonction des questions soulevées lors de l'analyse pétrographique, pour être soumis à une analyse chimique. Cette dernière a été réalisée suivant la méthode de spectrométrie de fluorescence X (WD-XRF, analyse dispersive en longueur d'onde). Elle permet de déterminer la composition des matières premières utilisées, et ainsi d'infirmer ou confirmer certaines suppositions. Les résultats sont traités par analyse factorielle des éléments identifiés dans les échantillons (Borgers, 2015).

\section{Les échantillons}

Les céramiques retenues proviennent de Bavay « Neuf Fontaines » ${ }^{\mathbf{1}}$, « rue de la Gare ${ }^{\mathbf{2}}$ et «Sablière Mathieu », ainsi que de Pont-sur$\mathrm{Sambre}^{\mathbf{3}}$ et de Famars-Technopôle [ill. 2]. Les ateliers de Bavay « Neuf Fontaines » et « rue de la Gare », à quelques centaines de mètres à peine, forment probablement une seule officine. Les deux produisent dès le début du $\mathrm{I}^{\mathrm{er}}$ siècle jusqu'aux années 70 de notre ère. Nous avons privilégié les mortiers estampillés par les potiers BVDE, ULPEFOX et BRARIATVS, un choix induit par la thématique de recherche du transfert technique entre Bavay et Pont-sur-Sambre. Les deux premiers potiers sont clairement liés à la production bavaisienne. Pour BRARIATVS, la question est plus complexe, puisque cette estampille a également été découverte au sein des rebuts de cuisson à Pont-sur-Sambre (Delmaire, 1972 ; Loridant et Ménard, 2002). Le choix des cinq échantillons de Bavay « rue de la Gare » s'est porté sur les cruches et pots de type Besançon, faute de mortiers estampillés pour cet atelier. Pour Pont-sur-Sambre, six mortiers, marqués par VARIATVS, VICTOR et BRARIATVS, ont été retenus. Les potiers VARIATVS et VICTOR ne sont attestés qu'au sein des rebuts de Pontsur-Sambre, bien qu'ils soient présents dans les contextes de consommation à Bavay. L'officine était active du dernier tiers du $\mathrm{I}^{\mathrm{er}}$ siècle jusqu'à la fin du $\mathrm{II}^{\mathrm{e}}$ ou le début du $\mathrm{III}^{\mathrm{e}}$ siècle. Cinq individus proviennent de trois des fours de FamarsTechnopôle, datés du milieu ou de la deuxième moitié du $\mathrm{II}^{\mathrm{e}}$ siècle (four 1335 et 1382) et de la première partie du III ${ }^{\mathrm{e}}$ siècle (four 5135). La différence chronologique est intéressante pour appréhender les questions d'évolution technique.

Sept échantillons concernent des matières premières. Trois proviennent de la carrière SECAB à Bellignies, située à $3 \mathrm{~km}$ au nord-ouest de Bavay ${ }^{4}$, et deux de Famars (un du Mont Houy et un des marnes de la Rhonelle) ${ }^{\mathbf{5}}$. Un dernier est un mélange de ces deux matériaux (éch. 30) [ill. 3].

\section{Les groupes pétrographiques \\ du groupe Bavay-Famars}

Pétrographiquement, quatre mélanges

différents sont reconnaissables : les pâtes 1, 2, 9 et 16 [ill. 4].

La pâte 1 se caractérise par une texture fine, contenant 15-20 \% de quartz et de glauconies, bien triés et parallèles à la surface, dans une argile calcaire. Les quartz, de type monocristallin et de grande taille ( $1 \mathrm{~mm}-\mathrm{O}, 2 \mathrm{~mm})$, sont parfois entourés d'oxydes de fer. Quelques autres rares dégraissants (chailles, micrites, foraminifères et stries ferrugineuses) sont présents. Les éléments de grande taille sont extrêmement calibrés et il existe peu de petites inclusions. La cuisson est réalisée à une température assez basse. Ce mélange est très proche de la sous-variante 1 de la pâte 9 (cf. infra). Il est utilisé pour la fabrication de mortiers de Bavay « Neuf Fontaines », notamment ceux estampillés par les potiers ULPEFOX et BVDE, sans aucun doute liés à l'officine du $\mathrm{I}^{\mathrm{er}}$ siècle (éch. 20 et 21). Deux mortiers du four 5135, daté de la première partie du III $^{\mathrm{e}}$ siècle, de FamarsTechnopôle (éch. 4 et 5) sont également produits avec le même mélange.

La pâte 2, proche de la 1, contient les mêmes éléments dans les mêmes proportions. La différence entre ces deux pâtes se matérialise par la présence de multiples petites inclusions (o,2$\mathrm{o}, 02 \mathrm{~mm}$ ), notamment quartz, micas, glauconies. La température de cuisson paraît plus haute que pour la 1. La pâte 2 est utilisée uniquement à Famars (éch. 1, 2 et 3) dans différents fours (1335, 1382, 5135 et 2995), du début de la production jusqu'au premier tiers du IV ${ }^{\mathrm{e}}$ siècle. Les gobelets bilobés dorés au mica du 1382 sont fabriqués dans une sous-variante plus épurée (éch. 2).

La pâte 9 (éch. 6 à 11) se distingue par $20 \%$ de grands quartz et foraminifères, bien triés et calibrés (1-0,2 mm), dans une argile calcaire. Les micrites, installées dans les vides et autour des inclusions, sont également importantes. D'autres dégraissants, en plus petite quantité, les accompagnent : la chaille, les glauconies, de rares grès, des oxydes de fer et de la matière végétale. Peu de petits éléments sont visibles. Une première sous-variante proche de la pâte 1 , utilisée pour la production des échantillons 6 (cruche) et 11 (pot) est fine, homogène et semble épurée. La deuxième sous-variante contient toujours de nombreux grands éléments, mais accompagnés de matières végétales brûlées 


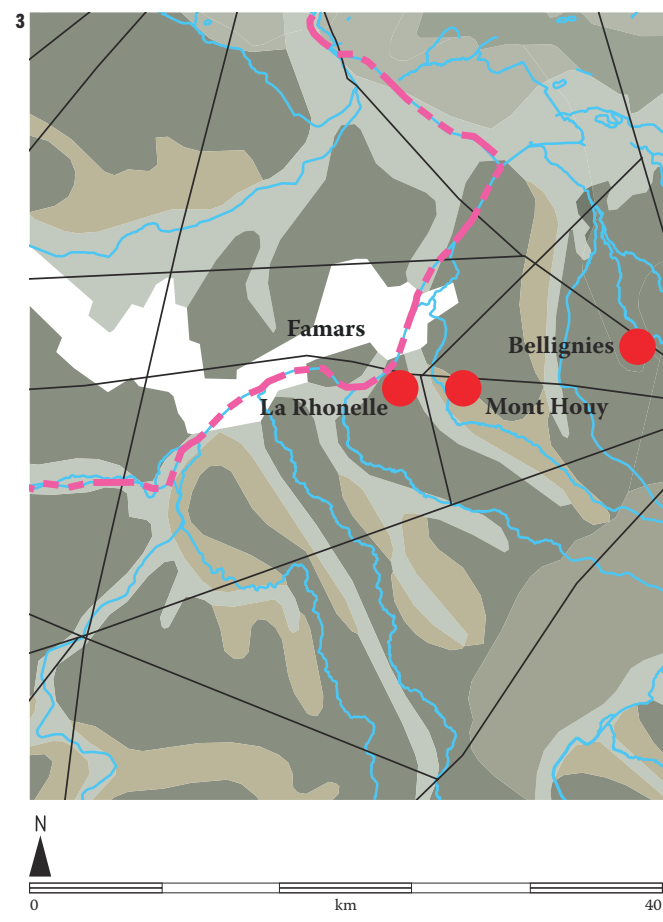

$\stackrel{1}{2}$
3. Localisation des gisements d'argile échantillonnés.

4. Les analyses réalisées

par Cécile Batigne avaient

démontré l'utilisation par

les potiers du nord de la Gaule

d'une argile fine contenant

peu d'inclusions et une teneur

de 9 à $13,5 \%$ de calcium

(Batigne-Vallet, 2001).

Ce constat est confirmé mais

les nouvelles recherches

montrent des différences

subtiles entre les trois sites

et au sein des ateliers.

Les pâtes 1 et 9 , utilisées

dans les ateliers de Bavay,

sont en général finement

granuleuses et évoluent vers

des pâtes plus fines à aspect

savonneux à Pont-sur-Sambre et Famars (pâtes 2 et 16).

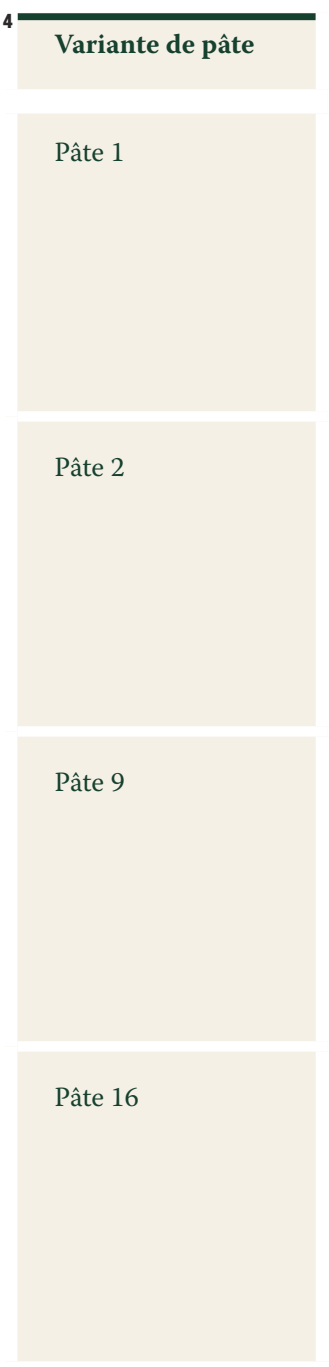

$\mathbf{N}^{\circ}$ échantillon
Échantillon 5
mortier à lèvre pendante

Famars four 5135, cruche à deux anses

Échantillon 3

Échantillon 9

Échantillon 17

Atelier

Bavay « rue de la Gare » céramique dorée au mica, US 1054

Pont-sur-Sambre,
Famars four 5135,

hoto macroscopique
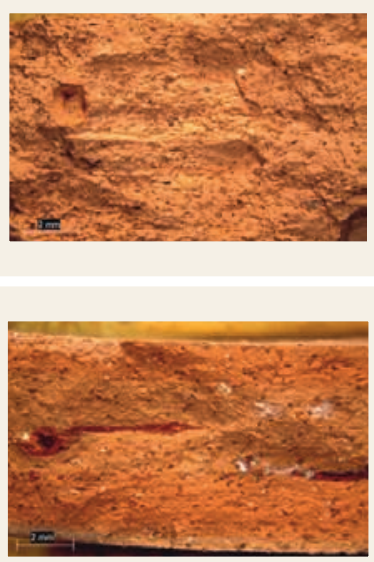
VARIATVS capital de cité

source d'argile échantillonnée

site mentionné

__ limites de la cité nervienne
Lame mince
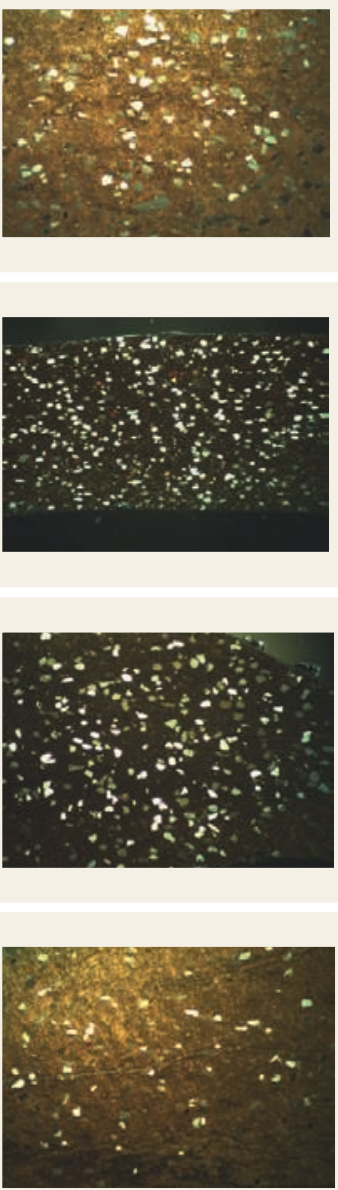
(éch. 10, pot à bord oblique). La température de cuisson en mode A paraît élevée. Cette pâte 9 est seulement utilisée dans l'officine de Bavay « rue de la Gare ».

La pâte 16 se caractérise par une matrice extrêmement épurée, d'aspect fin et doux, ou savonneux. Elle se distingue de la pâte 1 par la présence de seulement 5 à $10 \%$ de quartz et de glauconies de grande taille, moins calibrés et arrondis, ainsi que de micrites et foraminifères. Elle contient donc beaucoup moins d'inclusions que les variantes 1, 2 ou 9 dont elle est cependant proche. Les potiers ont plutôt choisi d'épurer/ décanter l'argile que de la tamiser puisque les inclusions de petite taille ont également disparu. La pâte 16 regroupe des échantillons de Bavay «Sablière Mathieu » (pot tonnelet, éch. 24), les deux mortiers estampillés par BRARIATVS de Bavay « Neuf Fontaines » (éch. 22 et 23), les cinq mortiers de Pont-sur-Sambre (éch. 14-18), ainsi que deux mortiers retrouvés dans les contextes de consommation de Famars-Technopôle, l'un estampillé par UXPVRO en pâte sableuse (éch. 12), et l'autre en pâte savonneuse du III ${ }^{\mathrm{e}}$ siècle (éch. 13).

Les échantillons d'argile tertiaire du Mont Houy et ceux provenant des marnes de Famars sont caractérisés par une minéralogie beaucoup plus fine que les céramiques de Famars-Technopôle. L'argile, de la carrière de Bellignies, est presque identique à la pâte des mortiers de Bavay « Neuf Fontaines » et de Pont-sur-Sambre.

\section{Les groupes chimiques}

Onze éléments majeurs et mineurs sont pris en compte pour l'analyse chimique : alumine $\left(\mathrm{Al}_{2} \mathrm{O}_{3}\right)$, baryum $(\mathrm{Ba})$, fer $\left(\mathrm{Fe}_{2} \mathrm{O}_{3}\right)$, potassium $\left(\mathrm{K}_{2} \mathrm{O}\right)$, magnésium $(\mathrm{MgO})$, strontium $(\mathrm{Sr})$, titane $\left(\mathrm{TiO}_{2}\right)$, silicium $\left(\mathrm{SiO}_{2}\right)$, calcium $(\mathrm{CaO})$, sodium $\left(\mathrm{Na}_{2} \mathrm{O}\right)^{2}$ et zirconium $(\mathrm{Zr})$. À partir des analyses XRF, une étude de la distribution met en évidence l'existence d'une ou plusieurs variantes de pâtes et d'éventuels problèmes de pollution et d'altération.

Les projections binaires et les classifications statistiques les complètent. Quatre diagrammes (relation strontium/calcium, calcium/alumine, fer/alumine et fer/magnésium) fournissent une image très homogène avec un groupement rectiligne pour la plupart des échantillons. Quelques variations se remarquent cependant, pour trois individus de Bavay « rue de la Gare » qui ont une teneur assez en haute en strontium et basse en calcium en contraste avec un exemple de Pont-sur-Sambre à ratio important en calcium. Ils se démarquent également par une proportion basse du magnésium dans le rapport fer/ magnésium, ou encore un pourcentage de baryum haut. Ces exceptions mises à part, la plupart des échantillons, aussi bien de Bavay « Neuf Fontaines », que de Pont-sur-Sambre ou de Famars-Technopôle, se caractérisent généralement par un ratio important en fer, alumine et titane, ainsi que par une teneur assez basse en baryum [ill. 5]. L'analyse par dendrogramme [ill. 6] souligne également la proximité des quatre variantes de pâtes distinguées pétrographiquement.

L'analyse chimique des matières premières les caractérise, hormis l'argile tertiaire du Mont Houy, comme des argiles à base calcaire, singularisées par une teneur assez basse pour les éléments suivants : fer $\left(\mathrm{Fe}_{2} \mathrm{O}_{3}\right)$, alumine $\left(\mathrm{Al}_{2} \mathrm{O}_{3}\right)$, potassium $\left(\mathrm{K}_{2} \mathrm{O}\right)$, magnésium $(\mathrm{MgO})$, strontium $(\mathrm{Sr})$ et zirconium $(\mathrm{Zr})$. Le dendrogramme évoque un lien plus proche entre les céramiques et l'argile de Bellignies contrairement aux marnes et à l'argile de FamarsMont Houy.

\section{L'interprétation des résultats}

Le fait que les variantes 1 et 16 se retrouvent dans plusieurs officines implique l'impossibilité de distinguer les lieux de production. Les différences sont minimes, car toutes les céramiques ont une base calcaire, comprenant les mêmes inclusions, mais en quantités différentes. La méthode intégrée est donc, dans ce cas, insuffisante, mais doit s'accompagner d'autres données, typologiques et chronologiques. Les analyses chimiques de matières premières suggèrent que les différentes officines ont toutes utilisé les mêmes argiles. Les ateliers de Bavay et de Pont-sur-Sambre, éloignés l'un de l'autre de seulement $10 \mathrm{~km}$, ont pu utiliser les mêmes bancs d'argile [ill. 7]. Famars, distante de Bavay de 20 km, n'a pas utilisé les marnes de la Rhonelle, et il n'est pas encore établi que, dans cette agglomération, les artisans aient disposé d'argiles aux mêmes caractéristiques. Des contrats de transports de matière première sont connus pour la région (Raepsaet, RaepsaetCharlier, 2007), et il faut considérer que les potiers de Famars ont pu importer les argiles calcaires.

Le dendrogramme démontre que les quatre groupes pétrographiques sont de composition proche. Ainsi, la pâte 1 est proche de la 16 , qui est à son tour similaire d'une sous-variante de la pâte 2. La 1 est aussi bien utilisée dans les ateliers de Bavay « Neuf Fontaines » (éch. 20-21) qu'à FamarsTechnopôle (éch. 4-5), et la 16 est utilisée à Bavay « Neuf Fontaines » (éch. 22-23), à Pont-sur-Sambre (éch. 14-18) et peut-être à Famars (éch. 12-13). L'utilisation de mêmes mélanges, et donc d'une technique identique, dans plusieurs officines et sur une période allant du $\mathrm{I}^{\mathrm{er}}$ siècle jusqu'au $\mathrm{III}^{\mathrm{e}}$ siècle indique des méthodes invariables. Il est manifeste que les potiers, malgré de petites différences dans le traitement, estiment que leurs mélanges sont au point et ne nécessitent pas d'adaptation ou d'amélioration. Les divergences, observées par exemple pour les ateliers de Bavay « rue de la Gare », indiquent certainement un groupe de potiers avec une habitude particulière, préférant des argiles à foraminifères. Leur répertoire ainsi que la période de production sont les mêmes qu'à Bavay « Neuf Fontaines », ce qui suggère simplement un choix. Cette analyse contraste nettement avec ce qui a été constaté pour la 


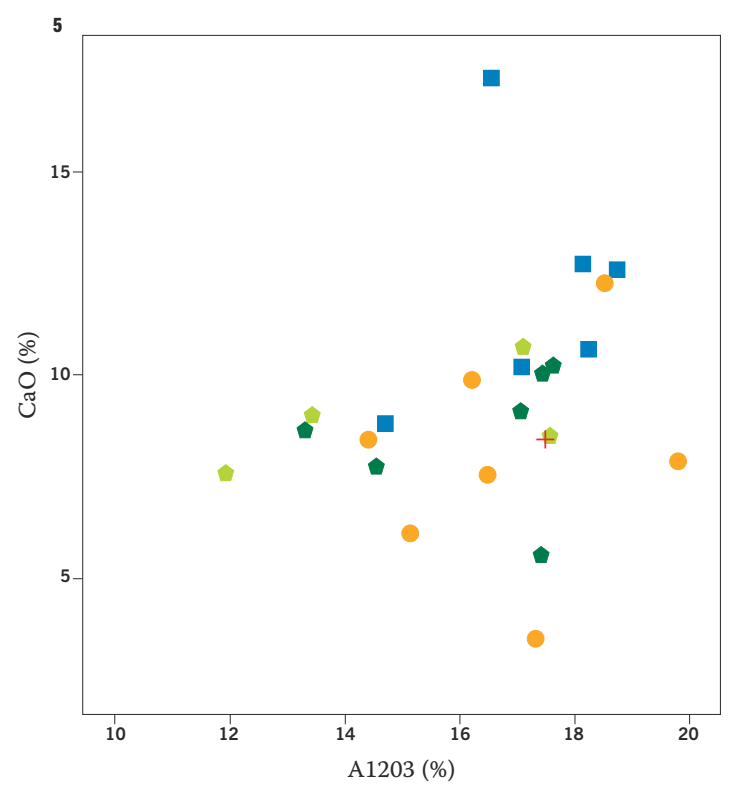

+ Bavay
Bavay Neuf Fontaines
- Bavay Rue de la Gare
- Famars
Pont-sur-Sambre 1989

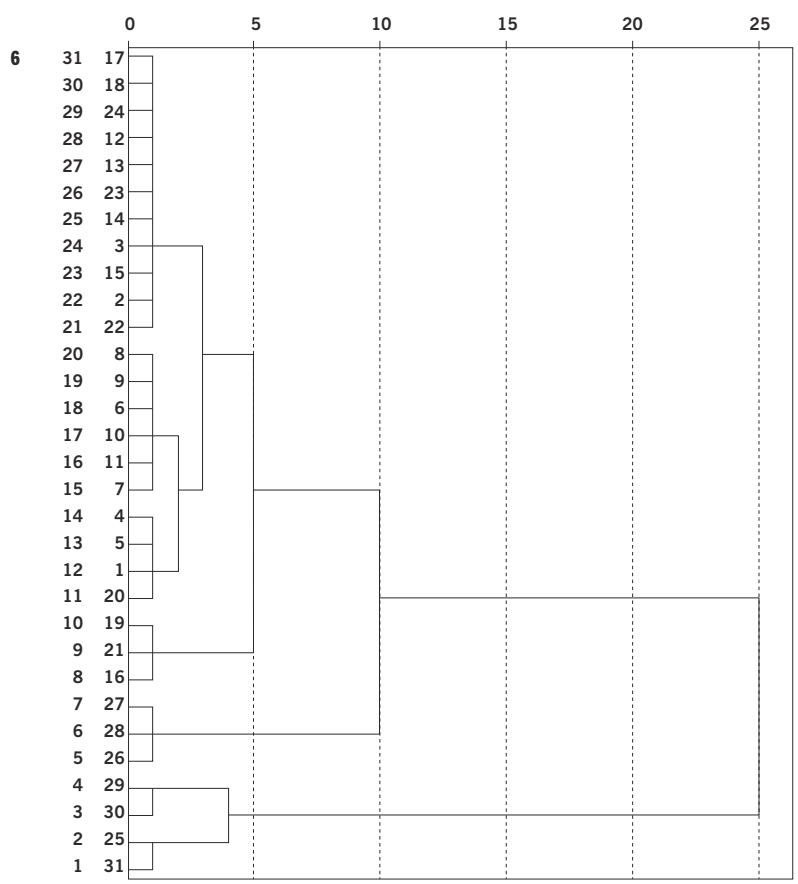

$\stackrel{\infty}{\infty}$

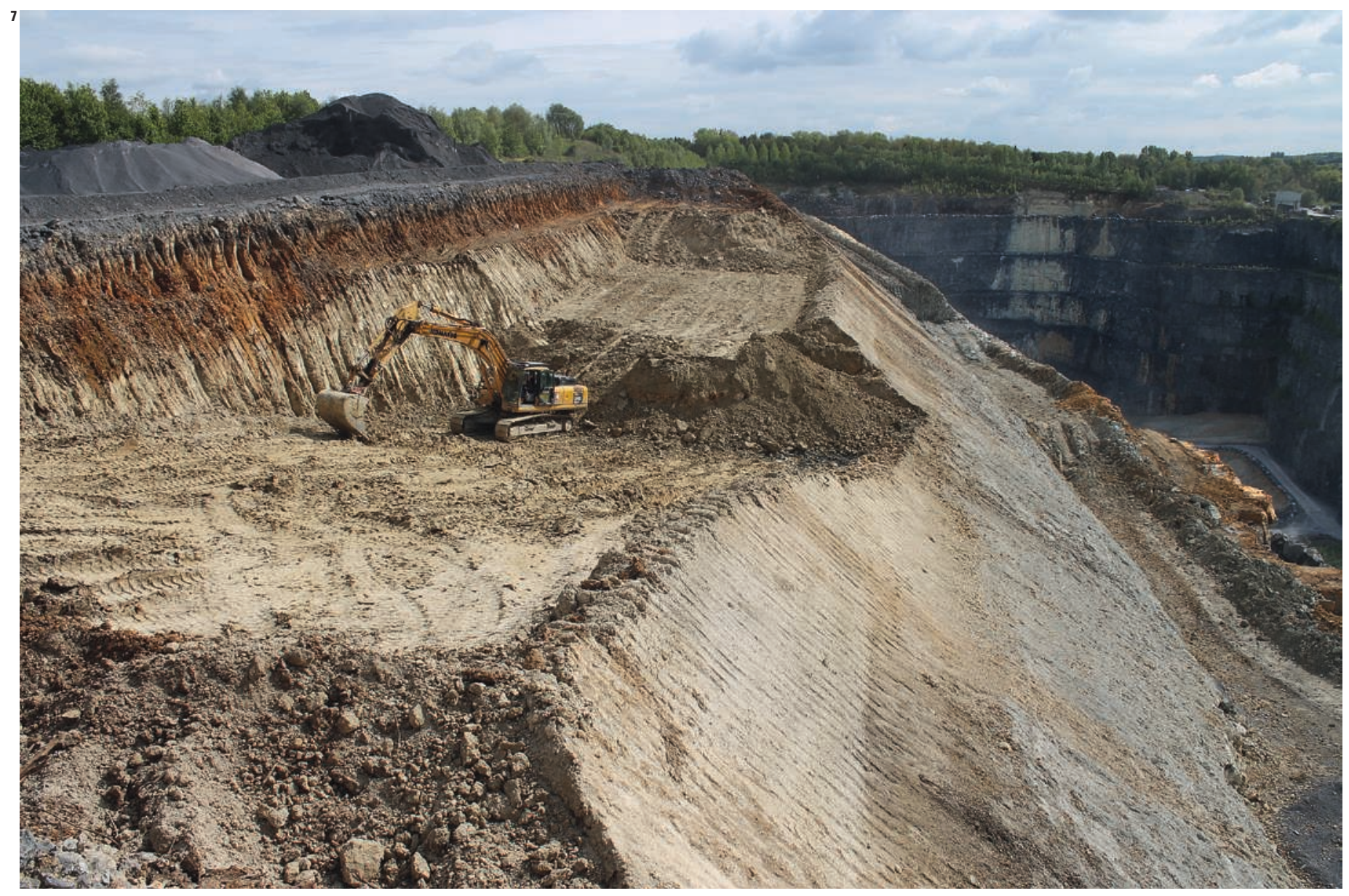




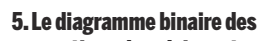

proportions de calcium et d'alumine illustre la proximité de toutes les pâtes, ainsi que l'apport haut en calcium pour certains échantillons de Pont-sur-Sambre. 6. Le dendrogramme regroupe l'analyse des mortiers de Pont-sur-Sambre, Famars, Bavay, estampillé par BRARIATVS, et le pot tonnelet. Il réunit les échantillons de Bavay « rue de la Gare " et établit le lien entre la pâte 1 de Bavay (éch. 20) et les cruches de Famars (éch. 4 et 5). Les argiles de Bellignies (éch. 26-28) sont plus proches des productions de Bavay que l'argile tertiaire du Mont Houy ou les marnes de la Rhonelle (éch. 29-31

et 25).

7. L'argile de la carrière de la SECAB à Bellignies es pétrographiquement et chimiquement la plus proche des échantillons analysés. céramique cuite en mode B à Famars-Technopôle (Willems, Borgers, 2016). Pour cette catégorie, une période de tests et des combinaisons évoluant pendant deux siècles aboutit, à la fin du $\mathrm{III}^{\mathrm{e}}$ siècle, à un mélange de haute qualité.

Les exemples de Bavay « Neuf Fontaines » sont extrêmement intéressants pour la compréhension de la délocalisation de l'officine vers Pont-surSambre et Famars. Son abandon est suggéré depuis longtemps par Frédéric Loridant (Loridant, 2001), mais n'a jamais été mis en évidence. La pâte 1 , utilisée par les potiers BVDE et ULPEFOX au $\mathrm{I}^{\text {er }}$ siècle (éch. 20-21), est retrouvée plus tard dans le four 5135 de Famars-Technopôle daté de la première partie du $\mathrm{III}^{\mathrm{e}}$ siècle. Bien que cet exemple souligne surtout un manque d'évolution, il implique également un transfert de techniques de génération en génération et renforce l'hypothèse d'une délocalisation non seulement à Pont-sur-Sambre, mais aussi à Famars.

La variante 16 employée par le potier BRARIATVS de Bavay « Neuf Fontaines » (éch. 22-23) est utilisée à Pont-sur-Sambre par VICTOR et VARIATVS au II ${ }^{\mathrm{e}}$ siècle (éch. 14-18) et est proche d'un échantillon légèrement différent produit par un potier du même nom de BRARIATVS (éch. 19). Cette similitude met en évidence la délocalisation de Bavay vers Pont-surSambre suggérée par Frédéric Loridant. La pâte 16 est d'ailleurs très apparentée à la 2 de Famars, utilisée pour la fabrication de cruches et de gobelets, qui en constitue probablement une variante. Il semble donc que le répertoire de Bavay ait été divisé entre ces deux nouvelles officines, les mortiers à Pont-sur-Sambre et les cruches à Famars. Un deuxième transfert a lieu au moment où l'agglomération de Pont-sur-Sambre arrête à son tour son activité. Famars reprend ensuite l'ensemble du répertoire, non seulement les cruches, mais aussi les mortiers, produits en pâte 1 et en variante 16 (éch. 12 et 13).

La méthode intégrée s'est avérée adaptée à la compréhension des évolutions techniques et de leurs transferts. La technique de traitement des argiles calcaires pour la fabrication d'un répertoire culinaire cuit en mode A évolue peu pendant plusieurs siècles. Les mélanges utilisés, considérés comme satisfaisants par les potiers, ne nécessitent aucune amélioration, ce qui n'entraîne que de rares évolutions dans la fabrication des cruches et mortiers. Les différentes variantes de pâtes traduisent dans ce cas le geste d'un potier ou d'un groupe de potiers et non une évolution ou amélioration technique. La présence de mêmes variantes au sein de plusieurs officines et à différentes époques suggère ici un transfert de personnes ou de techniques, et un maintien transgénérationnel des traditions.

L'utilisation d'une technique non évolutive implique ainsi que la pétrographie et la chimie ne permettent pas de distinction entre les officines, mais qu'elles doivent s'accompagner de données chronologiques et typologiques. Ceci contraste fortement avec ce qui a été constaté pour la céramique cuite en mode B de Famars-Technopôle, où les potiers cherchent à améliorer leurs mélanges pendant deux siècles, volonté démontrée par l'évolution complexe de mélanges et variantes abandonnés au cours du processus au lieu d'un maintien de traditions.

\section{Références bibliographiques}

Batigne-VAllet C., 2001, « Note sur les choix techniques attestés dans quelques ateliers de céramiques communes de Gaule du Nord pendant la période romaine », in SFECAG (éd.), Actes $d u$ congrès de Lille-Bavay, 24-27 mai 2001, Marseille, Société française d'étude de la céramique antique en Gaule, p. 207-208.

Borgers B., 2015, « Roman Pottery Production in the Civitas Tungrorum, Belgium: Towards an Integrated Compositional Approach », in GRUNwALd L. (éd.), Den Töpfern auf der Spur - Orte der

Keramikherstellung im Licht der neuesten Forschung, 46th International Symposium on Pottery, Mayen, 16-20 septembre 2013, p. 79-83.

Delmaire R., 1972, « Les mortiers de Pont-sur-Sambre et l'atelier de Brariatus. Contribution à l'étude de la céramique bavaisienne ", Septentrion, t. 2, fasc. 11-12, p. 46-54.
Dubois S., Willems S., Chaidron C., 2009, "Estampilles sur mortiers en céramique commune claire au chef-lieu des Ambiens », in SFECAG (éd.), Actes du congrès de Colmar, 21-24. mai 2009, Marseille, Société française d'étude de la céramique antique en Gaule, p. 677-688.

LORIDANT F., 2001, «Artisanat en milieu urbain : l'exemple des villes et des agglomérations secondaires du nord de la Gaule Belgique », in Polfer M. (éd.), L'artisanat romain : évolutions, continuités et ruptures (Italie et provinces occidentales), Actes du $2^{\mathrm{e}}$ colloque d'Erpeldange, 26-28 octobre 2001, Montagnac, M. Mergoil, «Monographies Instrumentum » 2O, p. 185-193. LORIDANT F., MÉNARd R., 2002, « Les mortiers dits "de Bavay". Une des productions de Pont-sur-Sambre (Nord) », in SFECAG (éd.), Actes du congrès de Bayeux, 9-12 mai 2002, Marseille, Société française d'étude de la céramique antique en Gaule, p. 431-435.

QUinn P.S., 2013, Ceramic Petrography: The interpretation of Archaeological Pottery and Related Artefacts in Thin Section, Oxford Archaeopress, $260 \mathrm{p}$
Raepsaet G., Raepsaet-Charlier M.-T., 2007, "Les briques et tuiles inscrites de Sains-du-Nord (Cité des Nerviens). Réflexions sur l'usage économique de l'écriture dans le monde galloromain ", L'Antiquité classique, t. 76, n 1, p. 133-148.

Willems S., 2005, Roman Pottery in the Tongeren Reference Collection : mortaria and coarse wares, Bruxelles, Vlaams Institut voor het Onroerend Erfgoed, $106 \mathrm{p}$

Willems S., Borgers B., 2015, « Comparaisons techniques des productions nerviennes: une première approche ", in SFECAG (éd.), Actes $d u$ congrès de Nyon, 14-17 mai 2015, Marseille, Société française d'étude de la céramique antique en Gaule, p. 273-284.

Willems S., Borgers B., 2016, « Pottery workshops at Fanum Martis (Northern France). Analysis of pottery production and consumption ", Acta Rei Cretariae Romanae Fautorum, 44, p. 429-437.

Picon M., 1973, Introduction à létude technique des céramiques sigillées de Lezoux, Dijon, Centre de recherche sur les techniques gréco-romaines, université de Dijon, 137 p. 